\title{
Supporting Information for \\ Near Attack Conformation as Strategy for ESIPT \\ Modulation for White-Light Generation
}

Emmanuel Santos Moraes, Luís Gustavo Teixeira Alves Duarte, José Carlos Germino and Teresa Dib Zambon Atvars*

Chemistry Institute, University of Campinas, Brazil.

*Corresponding Author.E-mail: tatvars@unicamp.br 


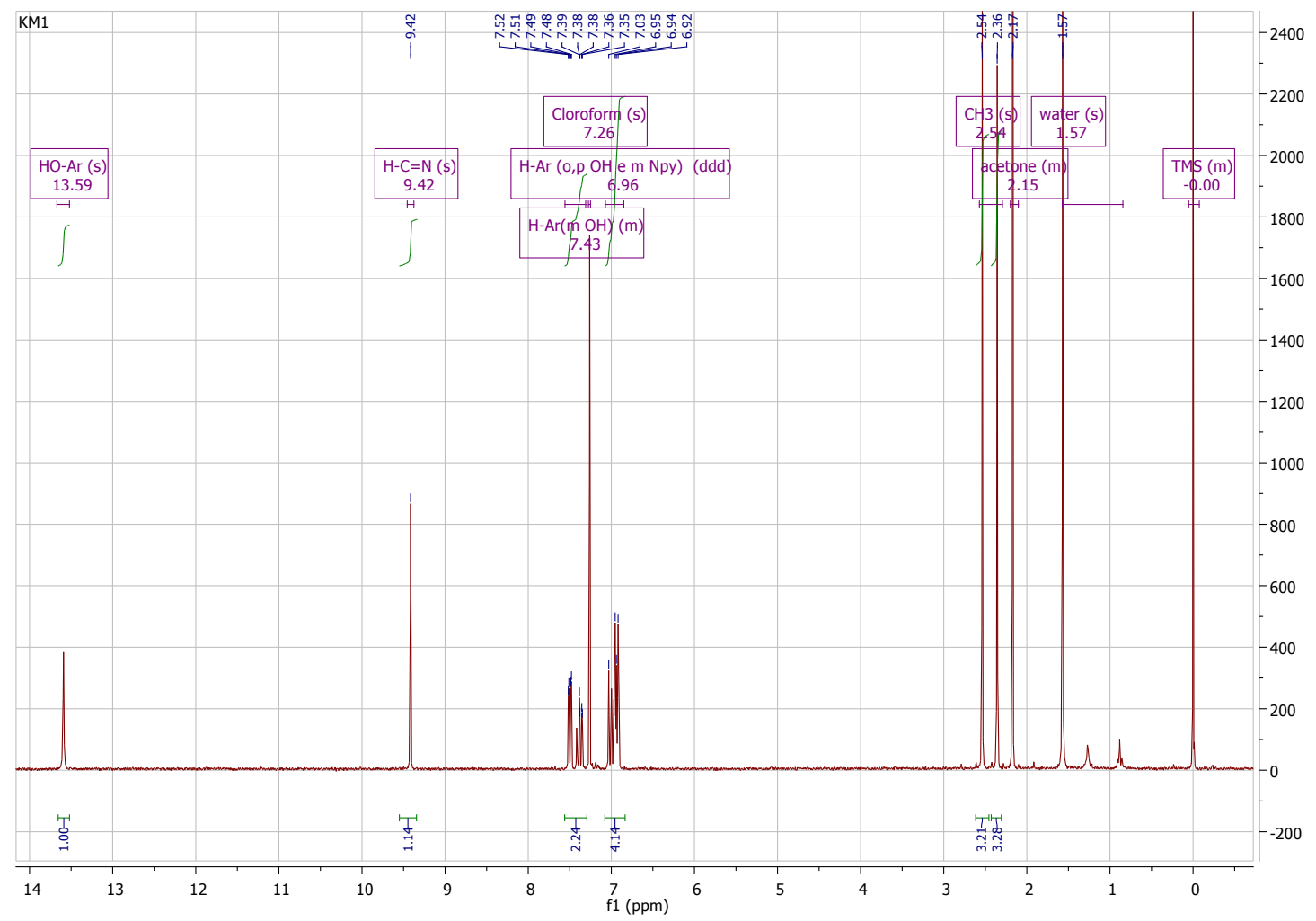

Figure S1. ${ }^{1} \mathrm{H}$ NMR spectrum in $\mathrm{CDCl}_{3}$ of $\mathrm{KM} 1$.

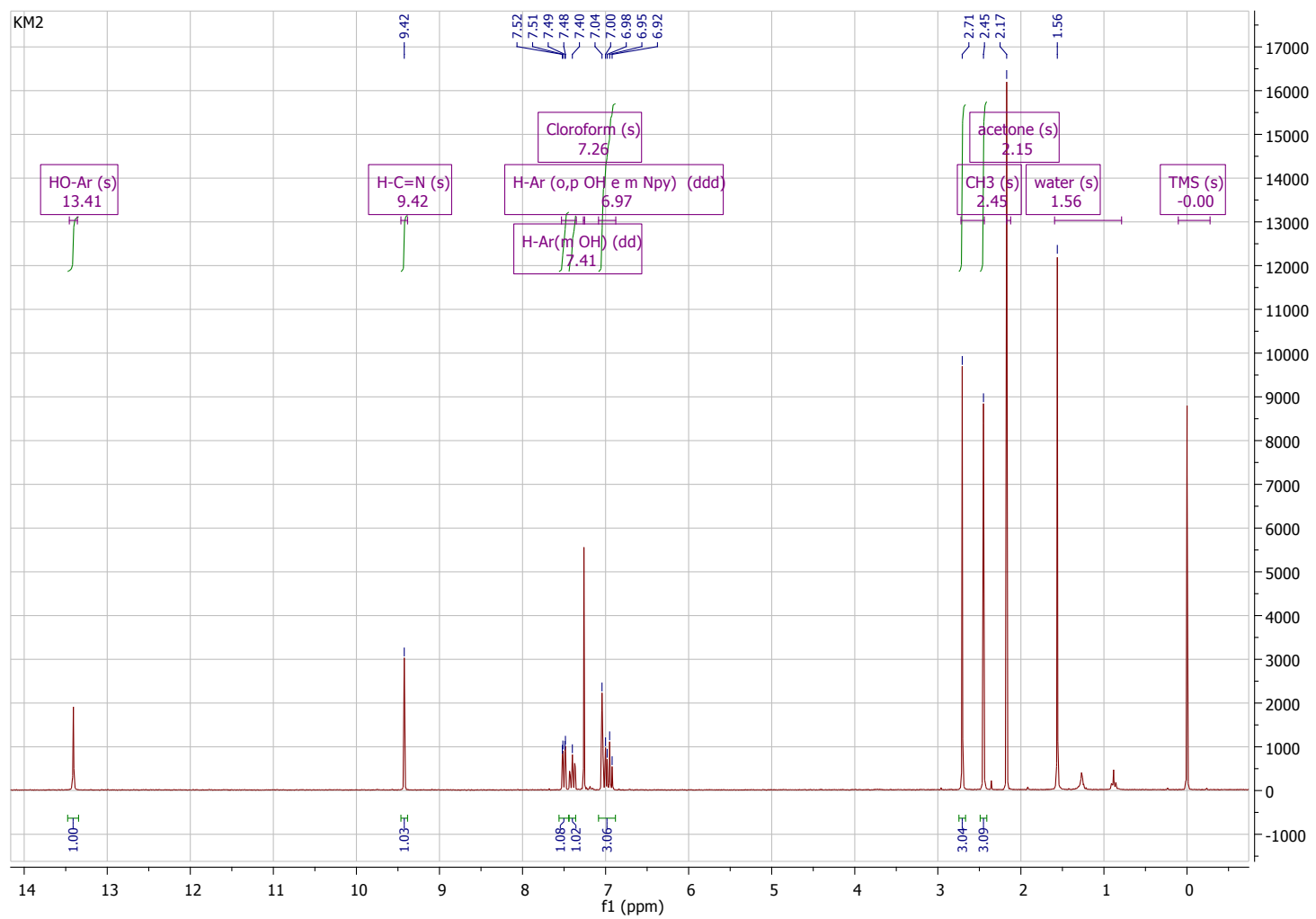

Figure S2. ${ }^{1} \mathrm{H}$ NMR spectrum in $\mathrm{CDCl}_{3}$ of $\mathrm{KM} 2$. 


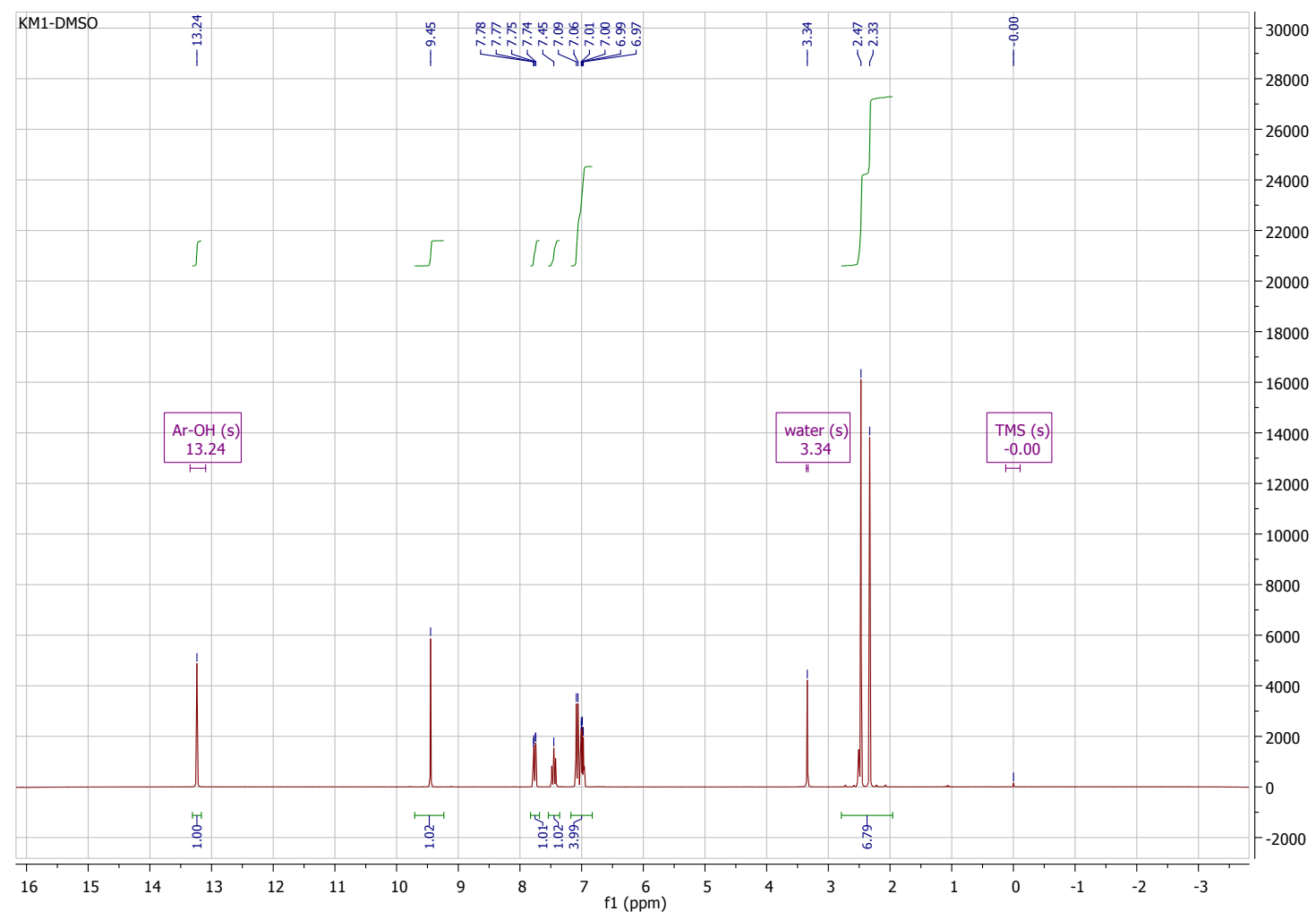

Figure S3. ${ }^{1} \mathrm{H}$ NMR spectrum in DMSO-d6 of KM1.

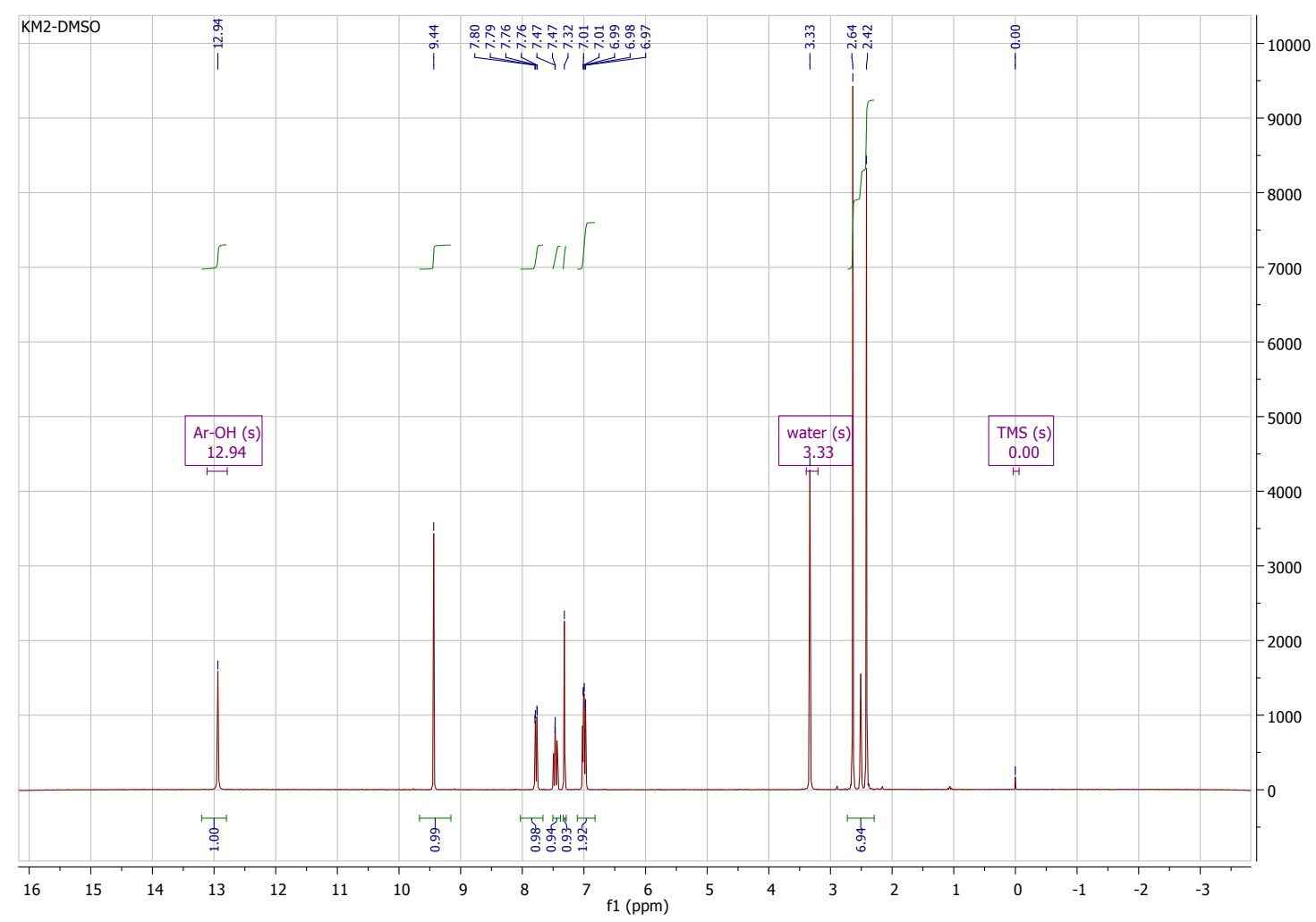

Figure S4. ${ }^{1} \mathrm{H}$ NMR spectrum in DMSO-d6 of KM2. 




Figure S5. ${ }^{13} \mathrm{C}$ NMR spectrum in DMSO- $d 6$ of KM1.

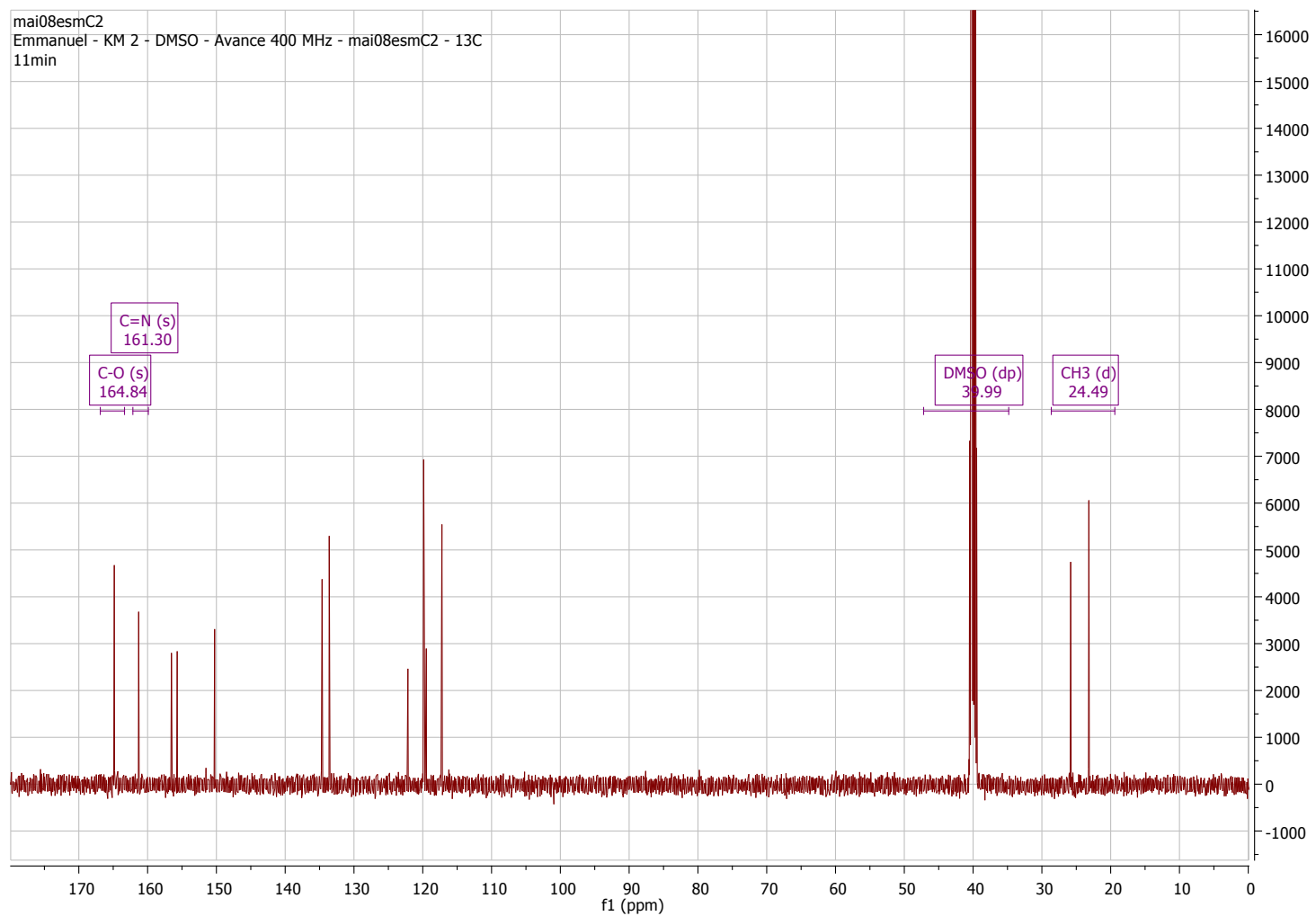

Figure S6. ${ }^{13} \mathrm{C}$ NMR spectrum in DMSO-d6 of KM2. 


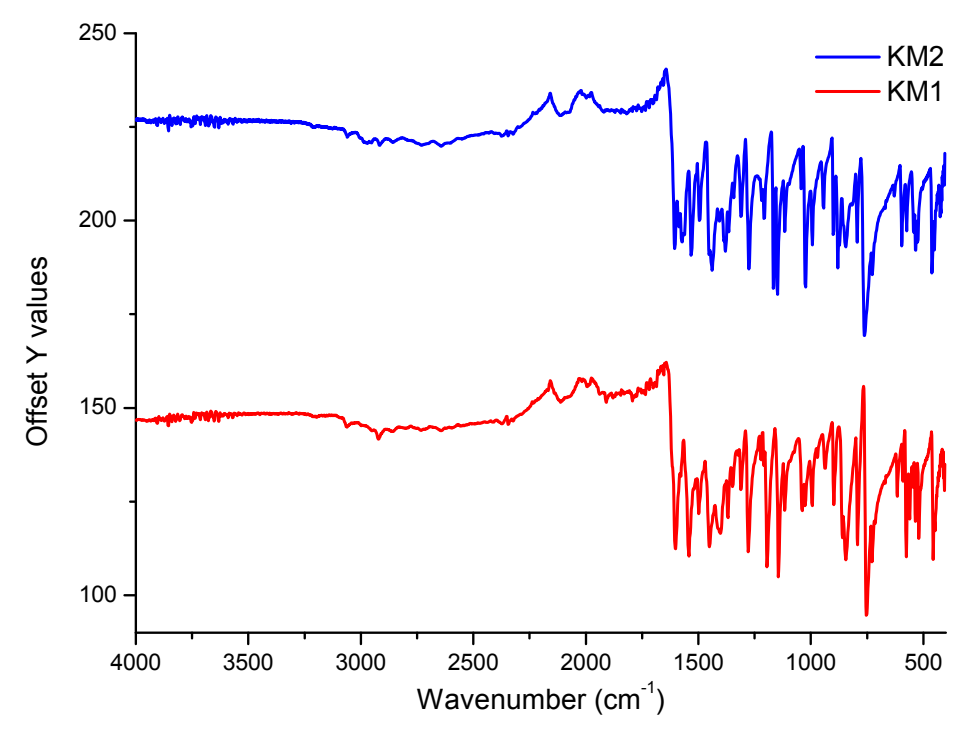

Figure S7. FTIR spectrum of KM1 (red line) and KM2 (blue line)

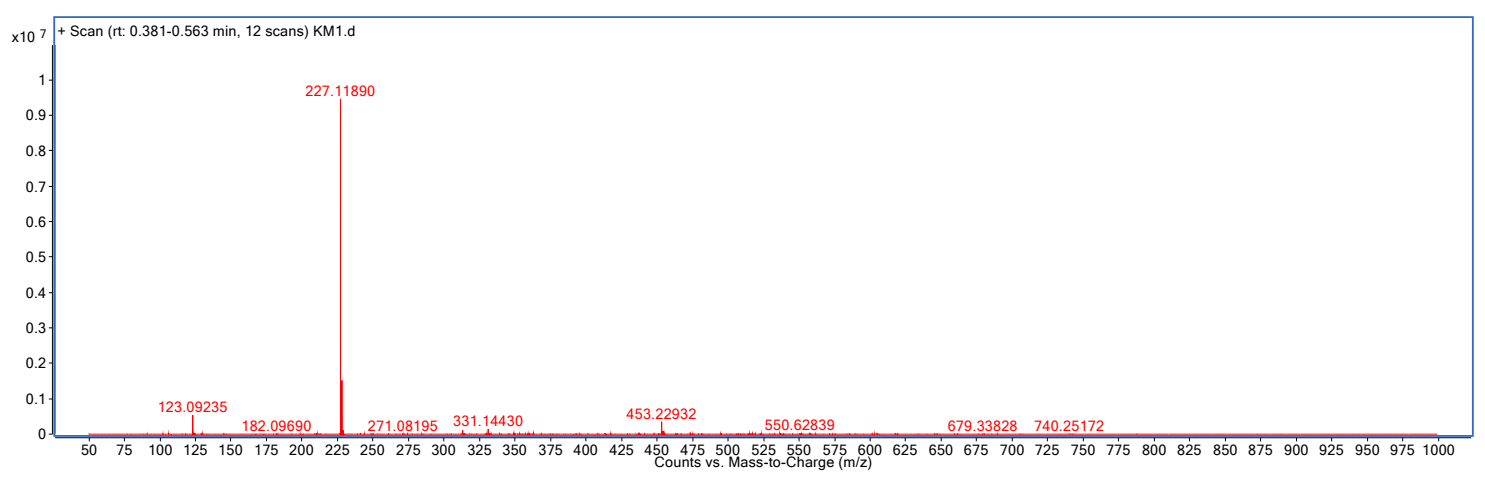

Figure S8. KM1 MS spectrum.

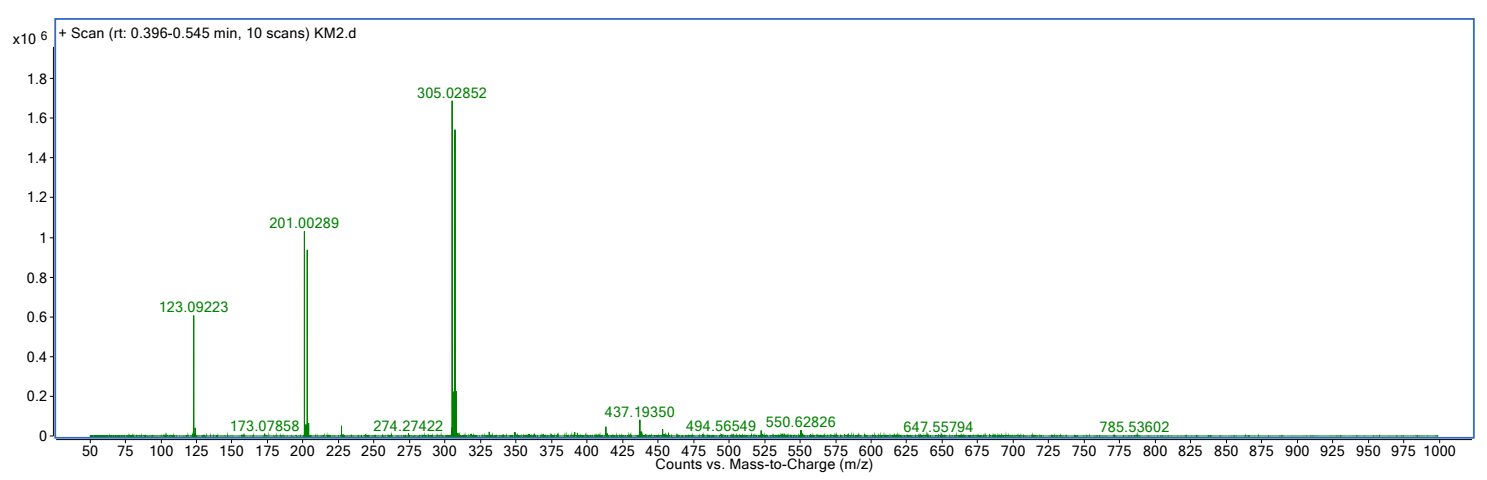

Figure S9. KM2 MS spectrum. 
Table S1. X-Ray Diffraction single Crystal data of KM1.

Fractional atomic coordinates and isotropic or equivalent isotropic displacement parameters $\left(\AA^{2}\right)$

\begin{tabular}{|c|c|c|c|c|}
\hline & $x$ & $y$ & $z$ & $U_{\text {iso }} * / U_{\text {eq }}$ \\
\hline O001 & $0.4550(3)$ & $0.2831(2)$ & $0.59416(8)$ & $0.0216(5)$ \\
\hline H001 & $0.573(6)$ & $0.333(4)$ & $0.5878(16)$ & $0.058(12)^{*}$ \\
\hline N002 & $1.0412(3)$ & $0.6667(2)$ & $0.61307(9)$ & $0.0176(5)$ \\
\hline N003 & $0.7623(3)$ & $0.4815(3)$ & $0.60348(9)$ & $0.0173(5)$ \\
\hline $\mathrm{C} 00 \mathrm{~A}$ & $1.2280(4)$ & $0.7215(3)$ & $0.59194(11)$ & $0.0173(6)$ \\
\hline $\mathrm{C} 00 \mathrm{~B}$ & $0.4014(4)$ & $0.5154(3)$ & $0.73734(12)$ & $0.0211(6)$ \\
\hline H00B & 0.4730 & 0.5954 & 0.7594 & $0.025 *$ \\
\hline $\mathrm{C} 00 \mathrm{C}$ & $0.6832(4)$ & $0.5262(3)$ & $0.65643(11)$ & $0.0184(6)$ \\
\hline $\mathrm{H} 00 \mathrm{C}$ & 0.7548 & 0.6037 & 0.6799 & $0.022 *$ \\
\hline C00D & $0.1902(4)$ & $0.2818(3)$ & $0.67326(12)$ & $0.0210(6)$ \\
\hline H00D & 0.1167 & 0.2023 & 0.6514 & $0.025^{*}$ \\
\hline $\mathrm{C} 00 \mathrm{E}$ & $0.2152(4)$ & $0.4555(3)$ & $0.76150(11)$ & $0.0232(6)$ \\
\hline H00E & 0.1587 & 0.4942 & 0.7998 & $0.028 *$ \\
\hline $\mathrm{COOF}$ & $1.3261(4)$ & $0.8525(3)$ & $0.62830(12)$ & $0.0234(6)$ \\
\hline H00A & 1.3506 & 0.9402 & 0.6001 & $0.035^{*}$ \\
\hline $\mathrm{H} 00 \mathrm{~F}$ & 1.4622 & 0.8189 & 0.6463 & $0.035^{*}$ \\
\hline $\mathrm{H} 00 \mathrm{G}$ & 1.2295 & 0.8838 & 0.6622 & $0.035^{*}$ \\
\hline $\mathrm{C} 00 \mathrm{G}$ & $0.1103(4)$ & $0.3377(3)$ & $0.72925(12)$ & $0.0223(6)$ \\
\hline $\mathrm{H} 00 \mathrm{H}$ & -0.0175 & 0.2955 & 0.7460 & $0.027 *$ \\
\hline $\mathrm{COOH}$ & $1.3517(4)$ & $0.4705(3)$ & $0.45051(13)$ & $0.0253(7)$ \\
\hline H00I & 1.3772 & 0.5518 & 0.4194 & $0.038^{*}$ \\
\hline H00J & 1.2622 & 0.3897 & 0.4319 & $0.038^{*}$ \\
\hline $\mathrm{H} 00 \mathrm{~K}$ & 1.4882 & 0.4258 & 0.4636 & $0.038^{*}$ \\
\hline $\mathrm{C} 004$ & $0.3785(4)$ & $0.3418(3)$ & $0.64874(11)$ & $0.0168(6)$ \\
\hline $\mathrm{C} 005$ & $0.9565(4)$ & $0.5473(3)$ & $0.58155(11)$ & $0.0171(6)$ \\
\hline $\mathrm{C} 006$ & $1.3307(4)$ & $0.6604(3)$ & $0.53964(12)$ & $0.0190(6)$ \\
\hline H006 & 1.4632 & 0.7024 & 0.5264 & $0.023 *$ \\
\hline $\mathrm{C} 007$ & $0.4871(4)$ & $0.4606(3)$ & $0.68077(11)$ & $0.0171(6)$ \\
\hline $\mathrm{C} 008$ & $1.2405(4)$ & $0.5382(3)$ & $0.50667(11)$ & $0.0180(6)$ \\
\hline
\end{tabular}




$\begin{array}{lllll}\text { C009 } & 1.0466(4) & 0.4813(3) & 0.52854(11) & 0.0174(6) \\ \text { H009 } & 0.9773 & 0.3987 & 0.5075 & 0.021^{*}\end{array}$

Atomic displacement parameters $\left(\AA^{2}\right)$

\begin{tabular}{|c|c|c|c|c|c|c|}
\hline & $U^{11}$ & $U^{22}$ & $U^{33}$ & $U^{12}$ & $U^{13}$ & $U^{23}$ \\
\hline O001 & $\begin{array}{l}0.0195 \\
(10)\end{array}$ & $\begin{array}{l}0.0216 \\
(10)\end{array}$ & $0.0236(9)$ & $-0.0002(9)$ & $0.0042(9)$ & $-0.0051(8)$ \\
\hline N002 & $\begin{array}{l}0.0177 \\
(11)\end{array}$ & $\begin{array}{l}0.0155 \\
(10)\end{array}$ & $\begin{array}{l}0.0196 \\
(11)\end{array}$ & $0.0009(10)$ & $-0.0015(9)$ & $0.0014(9)$ \\
\hline N003 & $\begin{array}{l}0.0149 \\
(10)\end{array}$ & $\begin{array}{l}0.0170 \\
(11)\end{array}$ & $\begin{array}{l}0.0199 \\
(10)\end{array}$ & $0.0021(10)$ & $-0.0001(9)$ & $0.0020(8)$ \\
\hline $\mathrm{C} 00 \mathrm{~A}$ & $\begin{array}{l}0.0171 \\
(14)\end{array}$ & $\begin{array}{l}0.0154 \\
(13)\end{array}$ & $\begin{array}{l}0.0193 \\
(11)\end{array}$ & $0.0006(11)$ & $\begin{array}{l}-0.0032 \\
(11)\end{array}$ & $0.0036(10)$ \\
\hline $\mathrm{C} 00 \mathrm{~B}$ & $\begin{array}{l}0.0202 \\
(14)\end{array}$ & $\begin{array}{l}0.0247 \\
(16)\end{array}$ & $\begin{array}{l}0.0185 \\
(12)\end{array}$ & 0.0008 (13) & $\begin{array}{l}-0.0034 \\
(11)\end{array}$ & $\begin{array}{l}-0.0001 \\
(12)\end{array}$ \\
\hline $\mathrm{C} 00 \mathrm{C}$ & $\begin{array}{l}0.0172 \\
(13)\end{array}$ & $\begin{array}{l}0.0186 \\
(14)\end{array}$ & $\begin{array}{l}0.0194 \\
(12)\end{array}$ & $0.0010(12)$ & $\begin{array}{l}-0.0032 \\
(11)\end{array}$ & $\begin{array}{l}-0.0001 \\
(11)\end{array}$ \\
\hline C00D & $\begin{array}{l}0.0189 \\
(14)\end{array}$ & $\begin{array}{l}0.0176 \\
(14)\end{array}$ & $\begin{array}{l}0.0266 \\
(13)\end{array}$ & $0.0000(12)$ & $\begin{array}{l}-0.0013 \\
(12)\end{array}$ & $0.0047(11)$ \\
\hline $\mathrm{C} 00 \mathrm{E}$ & $\begin{array}{l}0.0212 \\
(15)\end{array}$ & $\begin{array}{l}0.0332 \\
(16)\end{array}$ & $\begin{array}{l}0.0151 \\
(12)\end{array}$ & 0.0055 (14) & $0.0018(12)$ & $0.0029(11)$ \\
\hline $\mathrm{C} 00 \mathrm{~F}$ & $\begin{array}{l}0.0244 \\
(16)\end{array}$ & $\begin{array}{l}0.0224 \\
(15)\end{array}$ & $\begin{array}{l}0.0235 \\
(14)\end{array}$ & $\begin{array}{l}-0.0034 \\
(13)\end{array}$ & $\begin{array}{l}-0.0027 \\
(12)\end{array}$ & $\begin{array}{l}-0.0016 \\
(12)\end{array}$ \\
\hline $\mathrm{C} 00 \mathrm{G}$ & $\begin{array}{l}0.0179 \\
(14)\end{array}$ & $\begin{array}{l}0.0247 \\
(15)\end{array}$ & $\begin{array}{l}0.0244 \\
(14)\end{array}$ & 0.0003 & $0.0020(11)$ & $0.0081(13)$ \\
\hline $\mathrm{COOH}$ & $\begin{array}{l}0.0258 \\
(15)\end{array}$ & $\begin{array}{l}0.0232 \\
(15)\end{array}$ & $\begin{array}{l}0.0271 \\
(14)\end{array}$ & $\begin{array}{l}-0.0024 \\
(14)\end{array}$ & $0.0061(12)$ & $\begin{array}{l}-0.0026 \\
(13)\end{array}$ \\
\hline C004 & $\begin{array}{l}0.0166 \\
(13)\end{array}$ & $\begin{array}{l}0.0162 \\
(13)\end{array}$ & $\begin{array}{l}0.0176 \\
(12)\end{array}$ & $0.0051(12)$ & $0.0007(11)$ & $0.0029(11)$ \\
\hline $\mathrm{C} 005$ & $\begin{array}{l}0.0150 \\
(13)\end{array}$ & $\begin{array}{l}0.0168 \\
(13)\end{array}$ & $\begin{array}{l}0.0195 \\
(13)\end{array}$ & $\begin{array}{l}-0.0006 \\
(12)\end{array}$ & $\begin{array}{l}-0.0033 \\
(11)\end{array}$ & $0.0034(11)$ \\
\hline $\mathrm{C} 006$ & $\begin{array}{l}0.0155 \\
(14)\end{array}$ & $\begin{array}{l}0.0184 \\
(14)\end{array}$ & $\begin{array}{l}0.0231 \\
(13)\end{array}$ & $\begin{array}{l}-0.0009 \\
(12)\end{array}$ & $\begin{array}{l}-0.0020 \\
(11)\end{array}$ & $0.0044(12)$ \\
\hline $\mathrm{C} 007$ & $\begin{array}{l}0.0158 \\
(14)\end{array}$ & $\begin{array}{l}0.0193 \\
(13)\end{array}$ & $\begin{array}{l}0.0162 \\
(12)\end{array}$ & $0.0034(12)$ & $\begin{array}{l}-0.0028 \\
(10)\end{array}$ & $0.0030(11)$ \\
\hline $\mathrm{C} 008$ & $\begin{array}{l}0.0189 \\
(14)\end{array}$ & $\begin{array}{l}0.0154 \\
(13)\end{array}$ & $\begin{array}{l}0.0196 \\
(12)\end{array}$ & $0.0014(12)$ & $0.0002(11)$ & $0.0037(10)$ \\
\hline
\end{tabular}




$\begin{array}{lllllll}\mathrm{C} 009 & \begin{array}{l}0.0187 \\ (13)\end{array} & 0.0146 & 0.0189 & 0.0010(12) & \begin{array}{l}-0.0029 \\ (11)\end{array} & 0.0006(10)\end{array}$

Geometric parameters $\left(\AA,^{\circ}\right)$

\begin{tabular}{|c|c|c|c|}
\hline $\mathrm{O} 001-\mathrm{H} 001$ & $0.87(4)$ & $\mathrm{C} 00 \mathrm{~F}-\mathrm{H} 00 \mathrm{G}$ & 0.9800 \\
\hline $\mathrm{O} 001-\mathrm{C} 004$ & $1.356(3)$ & $\mathrm{C} 00 \mathrm{G}-\mathrm{H} 00 \mathrm{H}$ & 0.9500 \\
\hline $\mathrm{N} 002-\mathrm{C} 00 \mathrm{~A}$ & $1.342(3)$ & $\mathrm{COOH}-\mathrm{HOOI}$ & 0.9800 \\
\hline N002- $\mathrm{C} 005$ & $1.342(3)$ & $\mathrm{COOH}-\mathrm{H} 00 \mathrm{~J}$ & 0.9800 \\
\hline $\mathrm{N} 003-\mathrm{C} 00 \mathrm{C}$ & $1.291(3)$ & $\mathrm{COOH}-\mathrm{H} 00 \mathrm{~K}$ & 0.9800 \\
\hline $\mathrm{N} 003-\mathrm{C} 005$ & $1.423(3)$ & $\mathrm{C} 004-\mathrm{C} 00 \mathrm{D}$ & $1.391(3)$ \\
\hline $\mathrm{C} 00 \mathrm{~A}-\mathrm{C} 00 \mathrm{~F}$ & $1.505(4)$ & $\mathrm{C} 004-\mathrm{C} 007$ & $1.410(4)$ \\
\hline $\mathrm{COOB}-\mathrm{H} 00 \mathrm{~B}$ & 0.9500 & $\mathrm{C} 005-\mathrm{C} 009$ & $1.385(3)$ \\
\hline $\mathrm{C} 00 \mathrm{~B}-\mathrm{C} 00 \mathrm{E}$ & $1.377(4)$ & $\mathrm{C} 006-\mathrm{C} 00 \mathrm{~A}$ & $1.390(3)$ \\
\hline $\mathrm{C} 00 \mathrm{C}-\mathrm{H} 00 \mathrm{C}$ & 0.9500 & C006-H006 & 0.9500 \\
\hline $\mathrm{C} 00 \mathrm{D}-\mathrm{H} 00 \mathrm{D}$ & 0.9500 & $\mathrm{C} 006-\mathrm{C} 008$ & $1.390(4)$ \\
\hline $\mathrm{C} 00 \mathrm{D}-\mathrm{C} 00 \mathrm{G}$ & $1.381(4)$ & $\mathrm{C} 007-\mathrm{C} 00 \mathrm{~B}$ & $1.401(3)$ \\
\hline $\mathrm{C} 00 \mathrm{E}-\mathrm{H} 00 \mathrm{E}$ & 0.9500 & $\mathrm{C} 007-\mathrm{C} 00 \mathrm{C}$ & $1.450(4)$ \\
\hline $\mathrm{C} 00 \mathrm{E}-\mathrm{C} 00 \mathrm{G}$ & $1.395(4)$ & $\mathrm{C} 008-\mathrm{COOH}$ & $1.503(4)$ \\
\hline $\mathrm{C} 00 \mathrm{~F}-\mathrm{H} 00 \mathrm{~A}$ & 0.9800 & $\mathrm{C} 008-\mathrm{C} 009$ & $1.392(4)$ \\
\hline $\mathrm{C} 00 \mathrm{~F}-\mathrm{H} 00 \mathrm{~F}$ & 0.9800 & C009- $\mathrm{H} 009$ & 0.9500 \\
\hline $\mathrm{C} 004-\mathrm{O} 001-\mathrm{H} 001$ & $104(2)$ & $\mathrm{C} 00 \mathrm{E}-\mathrm{C} 00 \mathrm{G}-\mathrm{H} 00 \mathrm{H}$ & 119.7 \\
\hline $\mathrm{C} 00 \mathrm{~A}-\mathrm{N} 002-\mathrm{C} 005$ & $116.8(2)$ & $\mathrm{H} 00 \mathrm{I}-\mathrm{COOH}-\mathrm{H} 00 \mathrm{~J}$ & 109.5 \\
\hline $\mathrm{C} 00 \mathrm{C}-\mathrm{N} 003-\mathrm{C} 005$ & $119.7(2)$ & $\mathrm{H} 00 \mathrm{I}-\mathrm{COOH}-\mathrm{H} 00 \mathrm{~K}$ & 109.5 \\
\hline $\mathrm{N} 002-\mathrm{C} 00 \mathrm{~A}-\mathrm{C} 00 \mathrm{~F}$ & $116.8(2)$ & $\mathrm{H} 00 \mathrm{~J}-\mathrm{COOH}-\mathrm{H} 00 \mathrm{~K}$ & 109.5 \\
\hline $\mathrm{N} 002-\mathrm{C} 00 \mathrm{~A}-\mathrm{C} 006$ & $122.6(2)$ & $\mathrm{C} 008-\mathrm{C} 00 \mathrm{H}-\mathrm{H} 00 \mathrm{I}$ & 109.5 \\
\hline $\mathrm{C} 006-\mathrm{C} 00 \mathrm{~A}-\mathrm{C} 00 \mathrm{~F}$ & $120.6(2)$ & $\mathrm{C} 008-\mathrm{C} 00 \mathrm{H}-\mathrm{H} 00 \mathrm{~J}$ & 109.5 \\
\hline $\mathrm{C} 00 \mathrm{E}-\mathrm{C} 00 \mathrm{~B}-\mathrm{H} 00 \mathrm{~B}$ & 119.4 & $\mathrm{C} 008-\mathrm{C} 00 \mathrm{H}-\mathrm{H} 00 \mathrm{~K}$ & 109.5 \\
\hline $\mathrm{C} 00 \mathrm{E}-\mathrm{C} 00 \mathrm{~B}-\mathrm{C} 007$ & $121.2(3)$ & $\mathrm{O} 001-\mathrm{C} 004-\mathrm{C} 00 \mathrm{D}$ & $118.8(2)$ \\
\hline $\mathrm{C} 007-\mathrm{C} 00 \mathrm{~B}-\mathrm{H} 00 \mathrm{~B}$ & 119.4 & $\mathrm{O} 001-\mathrm{C} 004-\mathrm{C} 007$ & $121.1(2)$ \\
\hline $\mathrm{N} 003-\mathrm{C} 00 \mathrm{C}-\mathrm{H} 00 \mathrm{C}$ & 119.3 & $\mathrm{C} 00 \mathrm{D}-\mathrm{C} 004-\mathrm{C} 007$ & $120.1(2)$ \\
\hline $\mathrm{N} 003-\mathrm{C} 00 \mathrm{C}-\mathrm{C} 007$ & $121.4(2)$ & N002-C005-N003 & $118.9(2)$ \\
\hline $\mathrm{C} 007-\mathrm{C} 00 \mathrm{C}-\mathrm{H} 00 \mathrm{C}$ & 119.3 & $\mathrm{~N} 002-\mathrm{C} 005-\mathrm{C} 009$ & $124.3(2)$ \\
\hline $\mathrm{C} 00 \mathrm{G}-\mathrm{C} 00 \mathrm{D}-\mathrm{H} 00 \mathrm{D}$ & 120.0 & $\mathrm{C} 009-\mathrm{C} 005-\mathrm{N} 003$ & $116.9(2)$ \\
\hline $\mathrm{C} 00 \mathrm{G}-\mathrm{C} 00 \mathrm{D}-\mathrm{C} 004$ & $120.1(3)$ & $\mathrm{C} 00 \mathrm{~A}-\mathrm{C} 006-\mathrm{H} 006$ & 119.8 \\
\hline
\end{tabular}




$\begin{array}{lllll}\mathrm{C} 004-\mathrm{C} 00 \mathrm{D}-\mathrm{H} 00 \mathrm{D} & 120.0 & \mathrm{C} 008-\mathrm{C} 006-\mathrm{C} 00 \mathrm{~A} & 120.3(2) \\ \mathrm{C} 00 \mathrm{~B}-\mathrm{C} 00 \mathrm{E}-\mathrm{H} 00 \mathrm{E} & 120.3 & \mathrm{C} 008-\mathrm{C} 006-\mathrm{H} 006 & 119.8 \\ \mathrm{C} 00 \mathrm{~B}-\mathrm{C} 00 \mathrm{E}-\mathrm{C} 00 \mathrm{G} & 119.5(3) & \mathrm{C} 00 \mathrm{~B}-\mathrm{C} 007-\mathrm{C} 00 \mathrm{C} & 120.0(2) \\ \mathrm{C} 00 \mathrm{G}-\mathrm{C} 00 \mathrm{E}-\mathrm{H} 00 \mathrm{E} & 120.3 & \mathrm{C} 00 \mathrm{~B}-\mathrm{C} 007-\mathrm{C} 004 & 118.5(2) \\ \mathrm{C} 00 \mathrm{~A}-\mathrm{C} 00 \mathrm{~F}-\mathrm{H} 00 \mathrm{~A} & 109.5 & \mathrm{C} 004-\mathrm{C} 007-\mathrm{C} 00 \mathrm{C} & 121.6(2) \\ \mathrm{C} 00 \mathrm{~A}-\mathrm{C} 00 \mathrm{~F}-\mathrm{H} 00 \mathrm{~F} & 109.5 & \mathrm{C} 006-\mathrm{C} 008-\mathrm{C} 00 \mathrm{H} & 120.6(2) \\ \mathrm{C} 00 \mathrm{~A}-\mathrm{C} 00 \mathrm{~F}-\mathrm{H} 00 \mathrm{G} & 109.5 & \mathrm{C} 006-\mathrm{C} 008-\mathrm{C} 009 & 117.2(2) \\ \mathrm{H} 00 \mathrm{~A}-\mathrm{C} 00 \mathrm{~F}-\mathrm{H} 00 \mathrm{~F} & 109.5 & \mathrm{C} 009-\mathrm{C} 008-\mathrm{C} 00 \mathrm{H} & 122.2(2) \\ \mathrm{H} 00 \mathrm{~A}-\mathrm{C} 00 \mathrm{~F}-\mathrm{H} 00 \mathrm{G} & 109.5 & \mathrm{C} 005-\mathrm{C} 009-\mathrm{C} 008 & 118.9(2) \\ \mathrm{H} 00 \mathrm{~F}-\mathrm{C} 00 \mathrm{~F}-\mathrm{H} 00 \mathrm{G} & 109.5 & \mathrm{C} 005-\mathrm{C} 009-\mathrm{H} 009 & 120.6 \\ \mathrm{C} 00 \mathrm{D}-\mathrm{C} 00 \mathrm{G}-\mathrm{C} 00 \mathrm{E} & 120.6(3) & \mathrm{C} 008-\mathrm{C} 009-\mathrm{H} 009 & 120.6 \\ \mathrm{C} 00 \mathrm{D}-\mathrm{C} 00 \mathrm{G}-\mathrm{H} 00 \mathrm{H} & 119.7 & & & \end{array}$


Table S2. X-Ray Diffraction single crystal data of KM2.

Fractional atomic coordinates and isotropic or equivalent isotropic displacement parameters $\left(\AA^{2}\right)$

\begin{tabular}{|c|c|c|c|c|}
\hline & $x$ & $y$ & $z$ & $U_{\text {iso }} * / U_{\text {eq }}$ \\
\hline $\mathrm{Br} 01$ & $1.06447(3)$ & $0.66106(2)$ & $0.77052(2)$ & $0.03330(10)$ \\
\hline O002 & $0.5203(2)$ & $0.14756(14)$ & $0.52238(13)$ & $0.0315(4)$ \\
\hline H002 & $0.571(3)$ & $0.2005(9)$ & $0.5493(10)$ & $0.0472(6)^{*}$ \\
\hline N003 & $0.6329(2)$ & $0.34491(15)$ & $0.53577(15)$ & $0.0206(4)$ \\
\hline N004 & $0.7859(2)$ & $0.50974(15)$ & $0.53808(14)$ & $0.0197(4)$ \\
\hline $\mathrm{C} 00 \mathrm{~A}$ & $0.4333(2)$ & $0.3029(2)$ & $0.27669(18)$ & $0.0226(5)$ \\
\hline $\mathrm{H} 00 \mathrm{~A}$ & $0.4528(2)$ & $0.3728(2)$ & $0.24596(18)$ & $0.0271(6)^{*}$ \\
\hline $\mathrm{C} 00 \mathrm{~B}$ & $0.9191(2)$ & $0.5631(2)$ & $0.70005(18)$ & $0.0221(5)$ \\
\hline $\mathrm{C} 00 \mathrm{C}$ & $0.8499(2)$ & $0.4792(2)$ & $0.75809(18)$ & $0.0227(5)$ \\
\hline C00D & $0.5804(2)$ & $0.36333(18)$ & $0.43998(18)$ & $0.0197(5)$ \\
\hline H00D & $0.5991(2)$ & $0.43257(18)$ & $0.40780(18)$ & $0.0237(5)^{*}$ \\
\hline $\mathrm{C} 00 \mathrm{E}$ & $0.3782(3)$ & $0.0973(2)$ & $0.36454(19)$ & $0.0264(5)$ \\
\hline H00E & $0.3595(3)$ & $0.0267(2)$ & $0.39372(19)$ & $0.0317(6)^{*}$ \\
\hline $\mathrm{C} 00 \mathrm{~F}$ & $0.3471(3)$ & $0.2260(2)$ & 0.21902 (19) & $0.0265(5)$ \\
\hline $\mathrm{H} 00 \mathrm{~F}$ & $0.3067(3)$ & $0.2428(2)$ & 0.14914 (19) & $0.0318(6)^{*}$ \\
\hline $\mathrm{C} 00 \mathrm{G}$ & $0.3193(3)$ & $0.1236(2)$ & $0.26327(19)$ & $0.0266(5)$ \\
\hline H00G & $0.2589(3)$ & $0.0708(2)$ & 0.22345 (19) & $0.0319(6)^{*}$ \\
\hline $\mathrm{COOH}$ & $0.9412(3)$ & $0.6763(2)$ & $0.5291(2)$ & $0.0276(5)$ \\
\hline $\mathrm{H} 00 \mathrm{~b}$ & 0.9269 (19) & $0.6621(6)$ & $0.4522(2)$ & $0.0415(8)^{*}$ \\
\hline $\mathrm{H} 00 \mathrm{c}$ & $0.8797(14)$ & $0.7417(3)$ & $0.5486(11)$ & $0.0415(8)^{*}$ \\
\hline $\mathrm{H} 0 \mathrm{hh}$ & $1.0558(6)$ & $0.6882(9)$ & $0.5459(11)$ & $0.0415(8)^{*}$ \\
\hline $\mathrm{C} 00 \mathrm{I}$ & $0.8788(3)$ & $0.4638(2)$ & $0.87622(19)$ & $0.0354(6)$ \\
\hline H0Oi & $0.843(2)$ & $0.5292(7)$ & $0.9146(2)$ & $0.0532(9)^{*}$ \\
\hline $\mathrm{H} 00 \mathrm{j}$ & $0.8182(18)$ & $0.3999(9)$ & $0.9007(3)$ & $0.0532(9)^{*}$ \\
\hline $\mathrm{H} 00 \mathrm{k}$ & $0.9942(4)$ & $0.4521(16)$ & $0.8905(3)$ & $0.0532(9)^{*}$ \\
\hline $\mathrm{C} 005$ & $0.7244(2)$ & $0.42569(18)$ & $0.59178(17)$ & $0.0191(4)$ \\
\hline $\mathrm{C} 006$ & $0.4927(2)$ & 0.27977 (19) & $0.38027(17)$ & $0.0200(4)$ \\
\hline $\mathrm{C} 007$ & $0.8820(2)$ & $0.57998(19)$ & $0.59127(18)$ & $0.0210(5)$ \\
\hline $\mathrm{C} 008$ & $0.7490(2)$ & 0.40887 (19) & $0.70082(18)$ & $0.0207(4)$ \\
\hline
\end{tabular}




$\begin{array}{lllll}\text { H008 } & 0.6974(2) & 0.34983(19) & 0.73591(18) & 0.0248(5)^{*} \\ \text { C009 } & 0.4650(3) & 0.17512(19) & 0.42347(18) & 0.0220(5)\end{array}$

$\underline{\text { Atomic displacement parameters }\left(\AA^{2}\right)}$

\begin{tabular}{|c|c|c|c|c|c|c|}
\hline & $U^{11}$ & $U^{22}$ & $U^{33}$ & $U^{12}$ & $U^{13}$ & $U^{23}$ \\
\hline Br01 & $\begin{array}{l}0.02803 \\
(14)\end{array}$ & $\begin{array}{l}0.03394 \\
(17)\end{array}$ & $\begin{array}{l}0.03765 \\
(16)\end{array}$ & $\begin{array}{l}-0.00554 \\
(10)\end{array}$ & $\begin{array}{l}-0.00655 \\
(10)\end{array}$ & $\begin{array}{l}-0.01248 \\
(11)\end{array}$ \\
\hline $\mathrm{O} 002$ & $\begin{array}{l}0.0518 \\
(11)\end{array}$ & $0.0231(9)$ & $0.0194(9)$ & $-0.0056(8)$ & $-0.0045(7)$ & $0.0008(7)$ \\
\hline N003 & $0.0218(8)$ & $\begin{array}{l}0.0201 \\
(10)\end{array}$ & $0.0198(9)$ & $-0.0005(7)$ & $0.0003(7)$ & $-0.0023(8)$ \\
\hline N004 & $0.0199(8)$ & $\begin{array}{l}0.0194 \\
(10)\end{array}$ & $0.0196(9)$ & $0.0035(7)$ & $-0.0015(7)$ & $-0.0009(8)$ \\
\hline $\mathrm{C} 00 \mathrm{~A}$ & $\begin{array}{l}0.0208 \\
(10)\end{array}$ & $\begin{array}{l}0.0241 \\
(12)\end{array}$ & $\begin{array}{l}0.0229 \\
(11)\end{array}$ & $0.0022(9)$ & $-0.0004(8)$ & $0.0001(9)$ \\
\hline $\mathrm{C} 00 \mathrm{~B}$ & $0.0167(9)$ & $\begin{array}{l}0.0255 \\
(12)\end{array}$ & $\begin{array}{l}0.0240 \\
(11)\end{array}$ & $0.0012(8)$ & $-0.0030(8)$ & $-0.0065(9)$ \\
\hline $\mathrm{C} 00 \mathrm{C}$ & $\begin{array}{l}0.0195 \\
(10)\end{array}$ & $\begin{array}{l}0.0272 \\
(13)\end{array}$ & $\begin{array}{l}0.0213 \\
(11)\end{array}$ & $0.0044(9)$ & $-0.0011(8)$ & $\begin{array}{l}-0.0026 \\
(10)\end{array}$ \\
\hline C00D & $0.0186(9)$ & $\begin{array}{l}0.0200 \\
(12)\end{array}$ & $\begin{array}{l}0.0208 \\
(11)\end{array}$ & $0.0023(8)$ & $0.0018(8)$ & $-0.0008(9)$ \\
\hline $\mathrm{C} 00 \mathrm{E}$ & $\begin{array}{l}0.0303 \\
(12)\end{array}$ & $\begin{array}{l}0.0218 \\
(13)\end{array}$ & $\begin{array}{l}0.0274 \\
(13)\end{array}$ & $-0.0022(9)$ & $0.0050(10)$ & $\begin{array}{l}-0.0029 \\
(10)\end{array}$ \\
\hline $\mathrm{C} 00 \mathrm{~F}$ & $\begin{array}{l}0.0222 \\
(11)\end{array}$ & $\begin{array}{l}0.0336 \\
(14)\end{array}$ & $\begin{array}{l}0.0235 \\
(12)\end{array}$ & $0.0037(9)$ & $-0.0037(9)$ & $\begin{array}{l}-0.0042 \\
(10)\end{array}$ \\
\hline $\mathrm{C} 00 \mathrm{G}$ & $\begin{array}{l}0.0232 \\
(11)\end{array}$ & $\begin{array}{l}0.0292 \\
(13)\end{array}$ & $\begin{array}{l}0.0273 \\
(12)\end{array}$ & $-0.0020(9)$ & $-0.0016(9)$ & $\begin{array}{l}-0.0101 \\
(10)\end{array}$ \\
\hline $\mathrm{COOH}$ & $\begin{array}{l}0.0252 \\
(11)\end{array}$ & $\begin{array}{l}0.0217 \\
(12)\end{array}$ & $\begin{array}{l}0.0360 \\
(14)\end{array}$ & $0.0008(9)$ & $0.0015(9)$ & $0.0012(10)$ \\
\hline $\mathrm{COOI}$ & $\begin{array}{l}0.0376 \\
(13)\end{array}$ & $\begin{array}{l}0.0465 \\
(17)\end{array}$ & $\begin{array}{l}0.0219 \\
(13)\end{array}$ & $\begin{array}{l}-0.0031 \\
(12)\end{array}$ & $\begin{array}{l}-0.0059 \\
(10)\end{array}$ & $\begin{array}{l}-0.0010 \\
(12)\end{array}$ \\
\hline $\mathrm{C} 005$ & $0.0176(9)$ & $\begin{array}{l}0.0193 \\
(11)\end{array}$ & $\begin{array}{l}0.0204 \\
(11)\end{array}$ & $0.0036(8)$ & $-0.0002(8)$ & $-0.0031(9)$ \\
\hline $\mathrm{C} 006$ & $0.0180(9)$ & $\begin{array}{l}0.0214 \\
(12)\end{array}$ & $\begin{array}{l}0.0205 \\
(11)\end{array}$ & $0.0028(8)$ & $0.0010(8)$ & $-0.0038(9)$ \\
\hline $\mathrm{C} 007$ & $0.0171(9)$ & $\begin{array}{l}0.0195 \\
(11)\end{array}$ & $\begin{array}{l}0.0264 \\
(12)\end{array}$ & $0.0049(8)$ & $0.0017(8)$ & $-0.0027(9)$ \\
\hline
\end{tabular}




$\begin{array}{lllllll}\mathrm{C} 008 & \begin{array}{l}0.0200 \\ (10)\end{array} & \begin{array}{l}0.0206 \\ (12)\end{array} & \begin{array}{l}0.0215 \\ (11)\end{array} & 0.0019(8) & 0.0006(8) & 0.0002(9) \\ \mathrm{C} 009 & \begin{array}{l}0.0247 \\ (10)\end{array} & 0.0239 & 0.0176 & 0.0035(9) & 0.0020(8) & -0.0030(9)\end{array}$

Geometric parameters $\left(\AA,{ }^{\circ}\right)$

\begin{tabular}{|c|c|c|c|}
\hline $\mathrm{Br} 01-\mathrm{C} 00 \mathrm{~B}$ & $1.901(2)$ & $\mathrm{C} 00 \mathrm{G}-\mathrm{H} 00 \mathrm{G}$ & 0.9500 \\
\hline $\mathrm{O} 002-\mathrm{H} 002$ & 0.8400 & $\mathrm{COOH}-\mathrm{H} 00 \mathrm{~b}$ & 0.9800 \\
\hline $\mathrm{O} 002-\mathrm{C} 009$ & $1.351(3)$ & $\mathrm{COOH}-\mathrm{H} 00 \mathrm{c}$ & 0.9800 \\
\hline $\mathrm{N} 003-\mathrm{C} 00 \mathrm{D}$ & $1.284(3)$ & $\mathrm{COOH}-\mathrm{H} 00 \mathrm{~h}$ & 0.9800 \\
\hline $\mathrm{N} 003-\mathrm{C} 005$ & $1.420(3)$ & $\mathrm{C} 00 \mathrm{I}-\mathrm{H} 00 \mathrm{i}$ & 0.9800 \\
\hline N004-C005 & $1.332(3)$ & $\mathrm{COOI}-\mathrm{H} 00 \mathrm{j}$ & 0.9800 \\
\hline N004-C007 & $1.338(3)$ & $\mathrm{C} 00 \mathrm{I}-\mathrm{H} 00 \mathrm{k}$ & 0.9800 \\
\hline $\mathrm{COOA}-\mathrm{H} 00 \mathrm{~A}$ & 0.9500 & $\mathrm{C} 005-\mathrm{C} 008$ & $1.387(3)$ \\
\hline $\mathrm{C} 00 \mathrm{~A}-\mathrm{C} 00 \mathrm{~F}$ & $1.375(3)$ & $\mathrm{C} 006-\mathrm{C} 00 \mathrm{~A}$ & $1.402(3)$ \\
\hline $\mathrm{C} 00 \mathrm{~B}-\mathrm{C} 00 \mathrm{C}$ & $1.385(3)$ & $\mathrm{C} 006-\mathrm{C} 00 \mathrm{D}$ & $1.450(3)$ \\
\hline $\mathrm{C} 00 \mathrm{C}-\mathrm{C} 00 \mathrm{I}$ & $1.500(3)$ & $\mathrm{C} 006-\mathrm{C} 009$ & $1.407(3)$ \\
\hline COOD-H00D & 0.9500 & $\mathrm{C} 007-\mathrm{C} 00 \mathrm{~B}$ & $1.401(3)$ \\
\hline $\mathrm{C} 00 \mathrm{E}-\mathrm{H} 00 \mathrm{E}$ & 0.9500 & $\mathrm{C} 007-\mathrm{COOH}$ & $1.497(3)$ \\
\hline $\mathrm{C} 00 \mathrm{E}-\mathrm{C} 00 \mathrm{G}$ & $1.383(3)$ & $\mathrm{C} 008-\mathrm{C} 00 \mathrm{C}$ & $1.387(3)$ \\
\hline $\mathrm{C} 00 \mathrm{~F}-\mathrm{H} 00 \mathrm{~F}$ & 0.9500 & $\mathrm{C} 008-\mathrm{H} 008$ & 0.9500 \\
\hline $\mathrm{C} 00 \mathrm{~F}-\mathrm{C} 00 \mathrm{G}$ & $1.387(4)$ & $\mathrm{C} 009-\mathrm{C} 00 \mathrm{E}$ & $1.393(3)$ \\
\hline $\mathrm{C} 009-\mathrm{O} 002-\mathrm{H} 002$ & 109.5 & $\mathrm{H} 00 \mathrm{c}-\mathrm{COOH}-\mathrm{COO}$ & 109.5 \\
\hline $\mathrm{C} 00 \mathrm{D}-\mathrm{N} 003-\mathrm{C} 005$ & $120.30(19)$ & $\mathrm{H} 00 \mathrm{~h}-\mathrm{COOH}-\mathrm{H} 00 \mathrm{~b}$ & 109.5 \\
\hline $\mathrm{C} 007-\mathrm{N} 004-\mathrm{C} 005$ & $118.34(19)$ & $\mathrm{H} 0 \mathrm{Oh}-\mathrm{COOH}-\mathrm{H} 00 \mathrm{c}$ & 109.5 \\
\hline $\mathrm{H} 00 \mathrm{~A}-\mathrm{C} 00 \mathrm{~A}-\mathrm{C} 006$ & $119.55(13)$ & $\mathrm{H} 00 \mathrm{~h}-\mathrm{C} 00 \mathrm{H}-\mathrm{C} 007$ & 109.5 \\
\hline $\mathrm{C} 00 \mathrm{~F}-\mathrm{C} 00 \mathrm{~A}-\mathrm{H} 00 \mathrm{~A}$ & $119.55(14)$ & $\mathrm{H} 00 \mathrm{i}-\mathrm{C} 00 \mathrm{I}-\mathrm{C} 00 \mathrm{C}$ & 109.5 \\
\hline $\mathrm{C} 00 \mathrm{~F}-\mathrm{C} 00 \mathrm{~A}-\mathrm{C} 006$ & $120.9(2)$ & $\mathrm{H} 00 \mathrm{j}-\mathrm{COOI}-\mathrm{COOC}$ & 109.5 \\
\hline $\mathrm{C} 00 \mathrm{C}-\mathrm{C} 00 \mathrm{~B}-\mathrm{Br} 01$ & $119.28(17)$ & $\mathrm{H} 00 \mathrm{j}-\mathrm{C} 00 \mathrm{I}-\mathrm{H} 00 \mathrm{i}$ & 109.5 \\
\hline $\mathrm{C} 00 \mathrm{C}-\mathrm{C} 00 \mathrm{~B}-\mathrm{C} 007$ & $122.0(2)$ & $\mathrm{H} 00 \mathrm{k}-\mathrm{C} 00 \mathrm{I}-\mathrm{C} 00 \mathrm{C}$ & 109.5 \\
\hline $\mathrm{C} 007-\mathrm{C} 00 \mathrm{~B}-\mathrm{Br} 01$ & $118.71(17)$ & $\mathrm{H} 00 \mathrm{k}-\mathrm{C} 00 \mathrm{I}-\mathrm{H} 00 \mathrm{i}$ & 109.5 \\
\hline $\mathrm{C} 00 \mathrm{~B}-\mathrm{C} 00 \mathrm{C}-\mathrm{C} 008$ & $116.1(2)$ & $\mathrm{H} 00 \mathrm{k}-\mathrm{C} 00 \mathrm{I}-\mathrm{H} 00 \mathrm{j}$ & 109.5 \\
\hline $\mathrm{C} 00 \mathrm{I}-\mathrm{C} 00 \mathrm{C}-\mathrm{C} 00 \mathrm{~B}$ & $123.1(2)$ & N004-C005-N003 & $119.60(19)$ \\
\hline $\mathrm{C} 00 \mathrm{I}-\mathrm{C} 00 \mathrm{C}-\mathrm{C} 008$ & $120.7(2)$ & $\mathrm{C} 008-\mathrm{C} 005-\mathrm{N} 003$ & $116.5(2)$ \\
\hline
\end{tabular}




$\begin{array}{llll}\text { H00D-C00D-N003 } & 119.59(13) & \mathrm{C} 008-\mathrm{C} 005-\mathrm{N} 004 & 123.8(2) \\ \text { H00D-C00D-C006 } & 119.59(13) & \mathrm{C} 00 \mathrm{~A}-\mathrm{C} 006-\mathrm{C} 009 & 118.6(2) \\ \mathrm{C} 006-\mathrm{C} 00 \mathrm{D}-\mathrm{N} 003 & 120.8(2) & \mathrm{C} 00 \mathrm{D}-\mathrm{C} 006-\mathrm{C} 00 \mathrm{~A} & 119.6(2) \\ \mathrm{H} 00 \mathrm{E}-\mathrm{C} 00 \mathrm{E}-\mathrm{C} 009 & 120.22(14) & \mathrm{C} 00 \mathrm{D}-\mathrm{C} 006-\mathrm{C} 009 & 121.8(2) \\ \mathrm{C} 00 \mathrm{G}-\mathrm{C} 00 \mathrm{E}-\mathrm{H} 00 \mathrm{E} & 120.22(15) & \mathrm{C} 00 \mathrm{~B}-\mathrm{C} 007-\mathrm{N} 004 & 120.2(2) \\ \mathrm{C} 00 \mathrm{G}-\mathrm{C} 00 \mathrm{E}-\mathrm{C} 009 & 119.6(2) & \mathrm{C} 00 \mathrm{H}-\mathrm{C} 007-\mathrm{N} 004 & 116.4(2) \\ \mathrm{H} 00 \mathrm{~F}-\mathrm{C} 00 \mathrm{~F}-\mathrm{C} 00 \mathrm{~A} & 120.12(14) & \mathrm{C} 00 \mathrm{H}-\mathrm{C} 007-\mathrm{C} 00 \mathrm{~B} & 123.3(2) \\ \mathrm{C} 00 \mathrm{G}-\mathrm{C} 00 \mathrm{~F}-\mathrm{C} 00 \mathrm{~A} & 119.8(2) & \mathrm{C} 00 \mathrm{C}-\mathrm{C} 008-\mathrm{C} 005 & 119.3(2) \\ \mathrm{C} 00 \mathrm{G}-\mathrm{C} 00 \mathrm{~F}-\mathrm{H} 00 \mathrm{~F} & 120.12(13) & \mathrm{C} 00 \mathrm{C}-\mathrm{C} 008-\mathrm{H} 008 & 120.37(13) \\ \mathrm{C} 00 \mathrm{~F}-\mathrm{C} 00 \mathrm{G}-\mathrm{C} 00 \mathrm{E} & 120.9(2) & \mathrm{H} 008-\mathrm{C} 008-\mathrm{C} 005 & 120.37(13) \\ \mathrm{H} 00 \mathrm{G}-\mathrm{C} 00 \mathrm{G}-\mathrm{C} 00 \mathrm{E} & 119.54(15) & \mathrm{C} 00 \mathrm{E}-\mathrm{C} 009-\mathrm{O} 002 & 118.4(2) \\ \mathrm{H} 00 \mathrm{G}-\mathrm{C} 00 \mathrm{G}-\mathrm{C} 00 \mathrm{~F} & 119.54(13) & \mathrm{C} 00 \mathrm{E}-\mathrm{C} 009-\mathrm{C} 006 & 120.3(2) \\ \mathrm{H} 00 \mathrm{~b}-\mathrm{C} 00 \mathrm{H}-\mathrm{C} 007 & 109.5 & \mathrm{C} 006-\mathrm{C} 009-\mathrm{O} 002 & 121.4(2) \\ \mathrm{H} 00 \mathrm{c}-\mathrm{C} 00 \mathrm{H}-\mathrm{H} 00 \mathrm{~b} & 109.5 & & \end{array}$

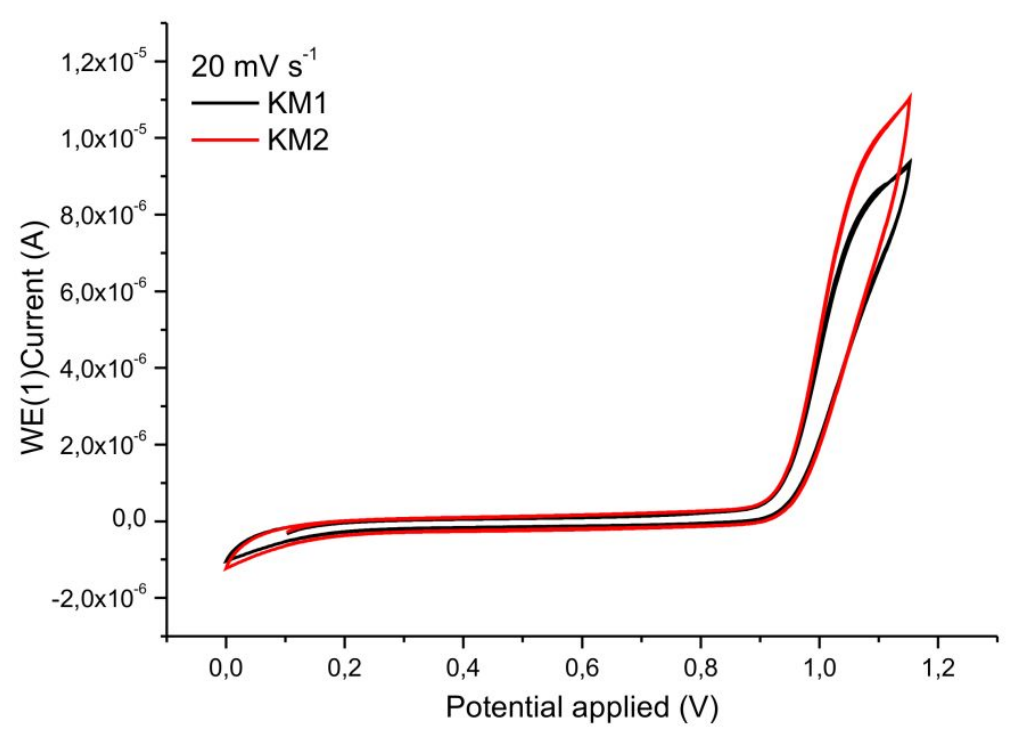

Figure S10. Cyclic Voltammograms of KM1 and KM2.

Table S3. The excitation energies $\lambda_{\text {abs }}(\mathrm{nm})$ and $\mathrm{f}$ oscillator strength, electronic transition assignment, $\lambda_{\exp }(\mathrm{nm})$ experimental absorption maxima, and $\theta_{\mathrm{C} 1-\mathrm{N} 2-\mathrm{C} 3-\mathrm{N} 4}$ the dihedral angle formed along C1-N2-C3-N4 at B3LYP/6-311+G* level in the gas phase and using CPCM model.

\begin{tabular}{|lccccc|}
\hline & $\lambda$ calc./nm & $\mathbf{f}$ & Assignment & $\lambda$ exp./nm & $\boldsymbol{\theta}_{\text {C1-N2-C3-N4 }}$ \\
\cline { 1 - 6 } Vacuum KM1 & $\mathbf{3 4 3}$ & 0,38 & H-L 87\% & 343 & $1^{\circ}$ \\
\hline
\end{tabular}






Figure S11. Normalized Experimental and theoretical spectra of KM1 and KM2 in different solvents.
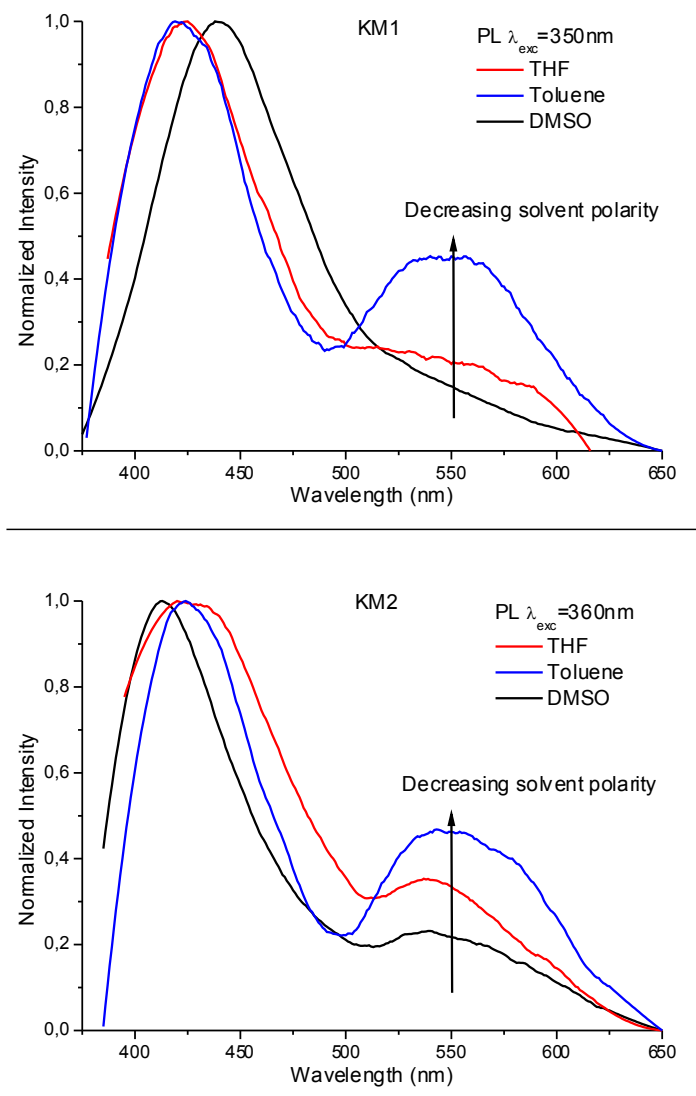

Figure S12. PL spectra of KM1 and KM2 in DMSO (black line), THF (red line) and Toluene (blue line). 



Figure S13. Normalized absorption and emission spectra of (a) KM1 and (b) KM2 in toluene solutions at $2.0 \mu \mathrm{mol} \mathrm{L}{ }^{-1}$. c) Frontier molecular orbitals diagram and relative energy among HOMO, LUMO, and LUMO+1 of KM1 $\mathrm{N}^{*}$ and $\mathrm{T}^{*}$ forms utilizing B3LYP/6-311+G*. 


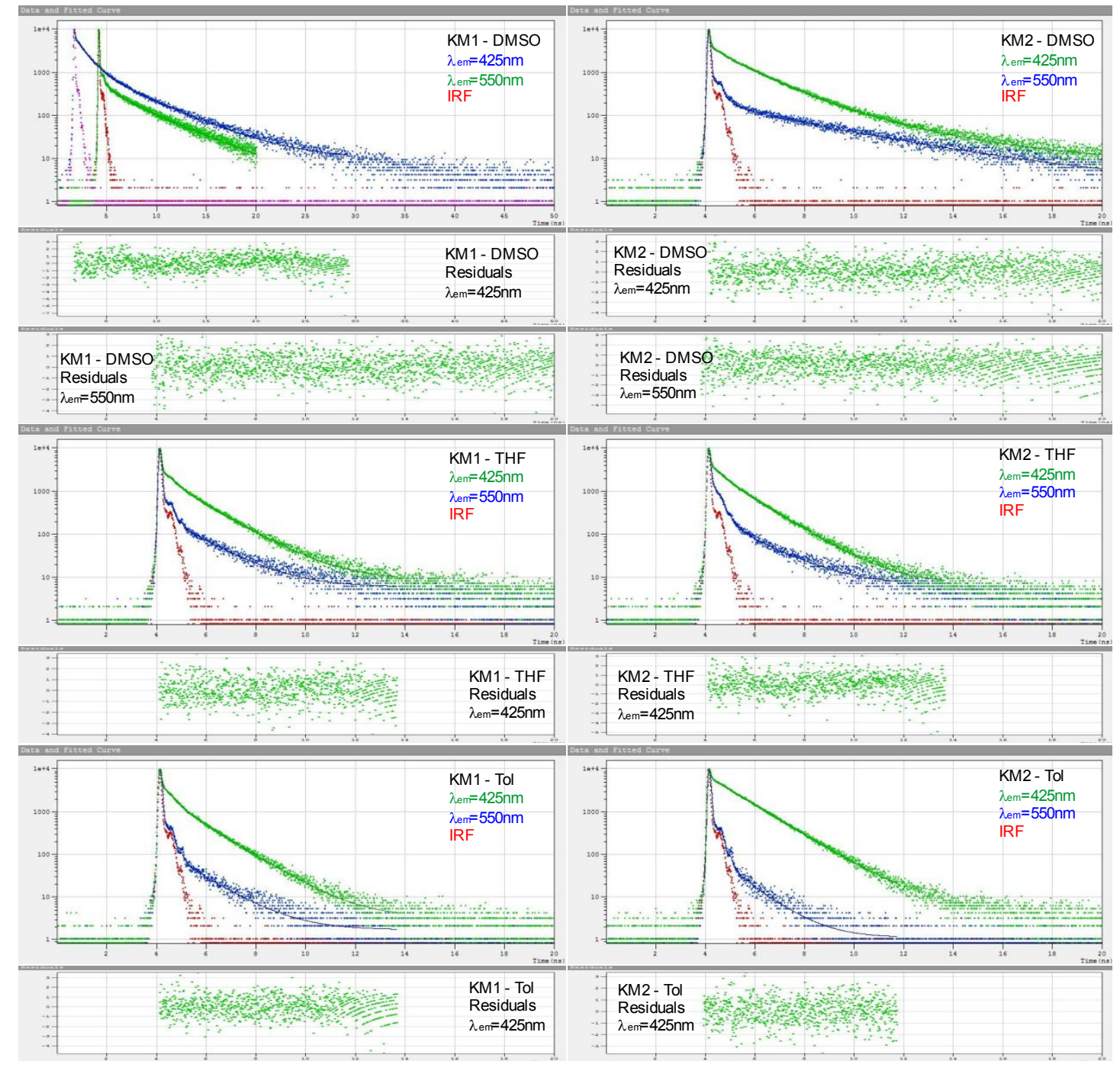

Figure S14. Fluorescence decays of KM1 and KM2. 


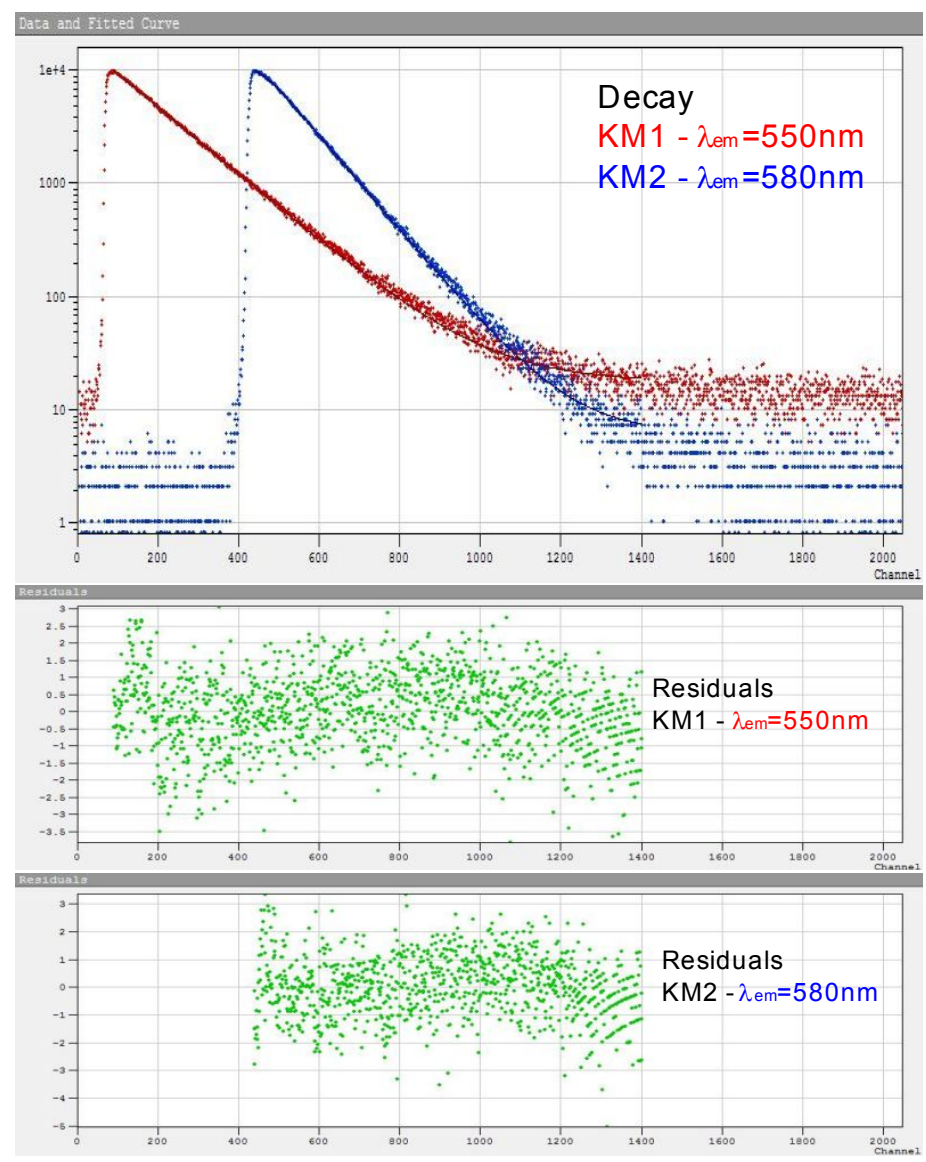

Figure S15. Fluorescence decays at 77K in vacuum for KM1 and KM2, respectively.

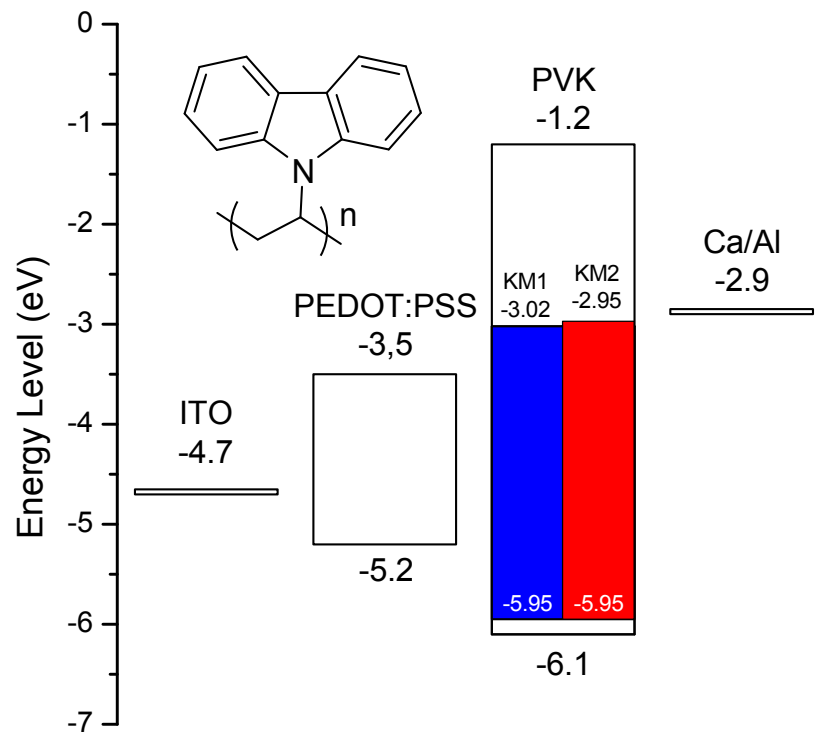

Figure S16. The diagram of energy levels for the charge transfer in OLEDs with the structure of polymer host (PVK). 




Figure S17. Compilation of the PL spectra in toluene $\lambda_{\text {exc }}=360 \mathrm{~nm}$ (black line), solidstate at $77 \mathrm{~K} \lambda_{\text {exc }}=370 \mathrm{~nm}$ (blue line), and EL spectra at $10 \mathrm{~V}$ (red line).

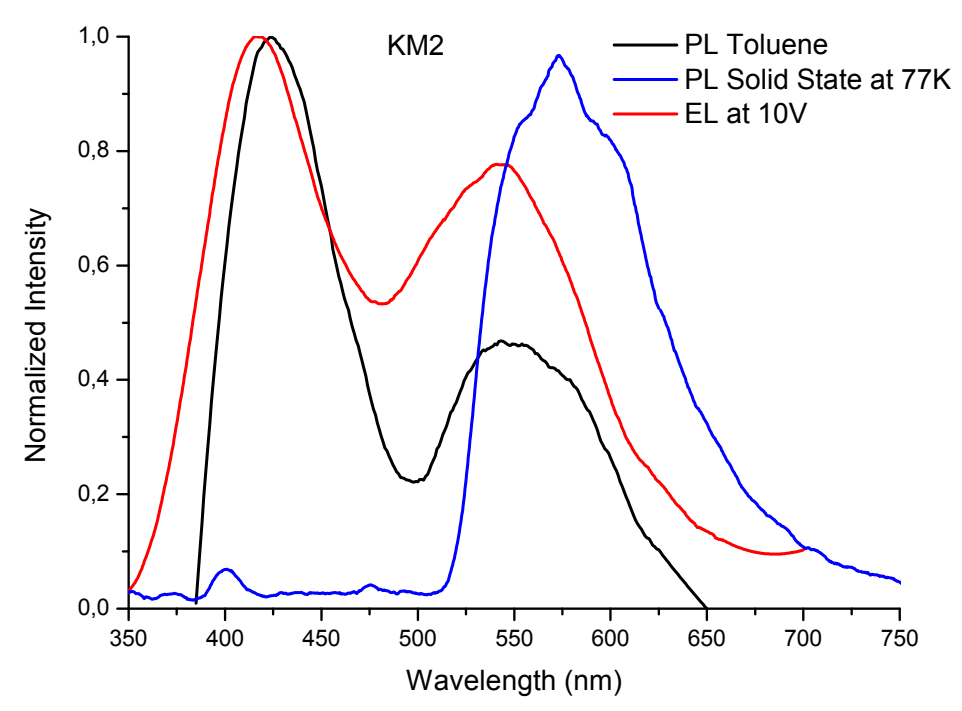

Figure S18. Compilation of the PL spectra in toluene $\lambda_{\mathrm{exc}}=350 \mathrm{~nm}$ (black line), solidstate at $77 \mathrm{~K} \lambda_{\text {exc }}=370 \mathrm{~nm}$ (blue line), and EL spectra at $10 \mathrm{~V}$ (red line). 


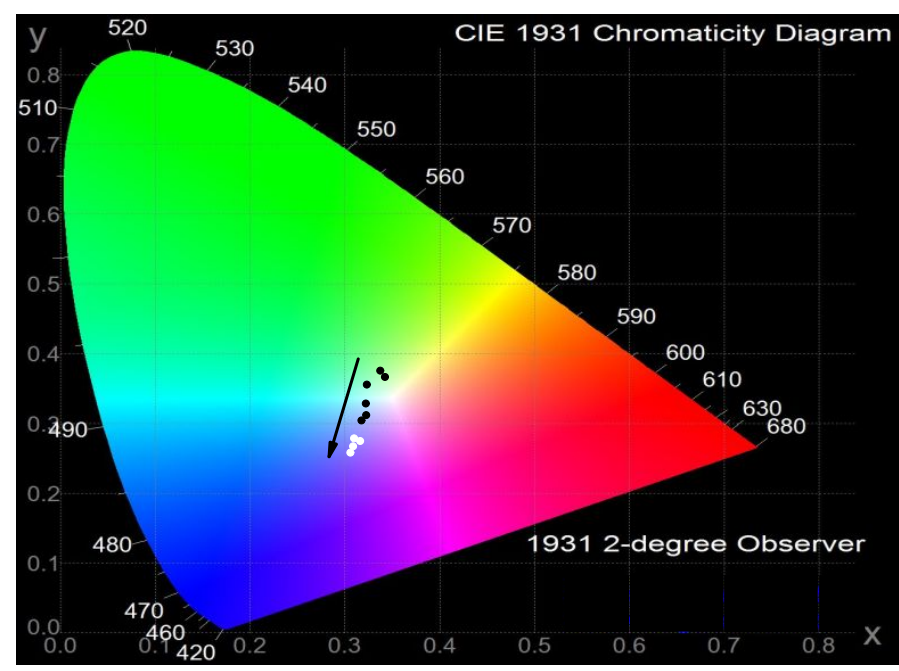

Figure S19. CIE1931 chromaticity diagram for KM1 (black circles) and KM2 (white circles) with the increase of voltage. 